\title{
INTEGRATED PROCESS MODELING FOR THE MECHANICAL PROPERTIES OPTIMIZATION OF DIRECT AGED ALLOY 718 ENGINE DISKS
}

\author{
Bernd Oberwinkler ${ }^{1}$, Andreas Fischersworring-Bunk ${ }^{2}$, Marco Hüller ${ }^{2}$, Martin Stockinger ${ }^{1}$ \\ ${ }^{1}$ Böhler Schmiedetechnik GmbH \& Co KG, Mariazellerstraße 25, Kapfenberg, 8605, Austria \\ ${ }^{2}$ MTU Aero Engines AG, Dachauer Straße 665, Munich, 80995, Germany
}

Keywords: Mechanical properties, Direct aging, Modeling, Yield strength, Creep behavior

\begin{abstract}
To meet the challenging requirements of future alloy 718 engine components, it is necessary to enable optimized processing and deliver forgings with tailored properties. In this paper, an integrated process modeling procedure is presented which is able to predict mechanical properties such as yield strength, low cycle fatigue and creep behavior of direct aged alloy 718 forgings. It includes thermo-mechanical process modeling in DEFORM ${ }^{\mathrm{TM}}$ $\left(\right.$ DEFORM $^{\mathrm{TM}}$ is a registered trademark of Scientific Forming Technologies Corporation), thermo-kinetic precipitation modeling in MatCalc, mechanical properties post-processing in BoMePP and visualization in ParaView. With respect to yield strength modeling, the following six strengthening contributions of direct aged alloy 718 are discussed in detail: basic yield strength, strain hardening, grain boundary strengthening, precipitation strengthening, solid solution strengthening, and the direct age effect. Furthermore, a relative importance of these strengthening contributions is presented. Verification of the integrated process modeling procedure and the according optimization potential for alloy 718 engine disks are examined on the basis of experimental results.
\end{abstract}

\section{Introduction}

Alloy 718 has been a workhorse nickel-base superalloy since the late 1960's [1]. Its strength and balanced mechanical properties at relatively low cost combined with its corrosion resistance and processing capabilities paved its way to many applications, especially for critical aerospace components like engine mounts and turbine disks. However, the continuing demand for higher efficiencies and lightweight design in aerospace pushes alloy 718 to its limits. The temperatures of many components in new engines are steadily increasing [1], which necessitates either an optimized utilization of conventional alloys like 718 or an introduction of new advanced superalloys. As the latter is costly and time consuming, alloy 718 will be the predominating superalloy for critical rotating gas turbine components at least for the next 10 years to come [2]. To enable optimized processing and deliver tailored properties to meet the challenging requirements of future engine components, it is essential to computationally model alloy 718 processing and its resulting mechanical properties. The latter are highly influenced by both the chemistry of the material and by microstructural features. These microstructural influences are the grain size, the type, size, morphology, fraction and distribution of precipitates, and the dislocation density and arrangement. Grain size modeling - for example, based on semiempirical models [3] - has been state-of-the-art for several years. Yet, besides grain boundary strengthening, precipitation strengthening has a major impact on the mechanical properties. Therefore, the thermo-kinetic modeling of precipitation in alloy 718 [4] is an important basis for the optimization of its processing with respect to precipitation strengthening.
Finally, the dislocation density and the according strain hardening [5] will have an impact on mechanical properties. Using these parameters as an input, the local yield strength can be modeled. Incorporating such information in the forging process-design guarantees a robust thermo-mechanical processing and optimized engine disks with tailored properties. The focus of this research was on direct aged alloy 718. Strengthening contributions, mechanical properties modeling and verification will be discussed below.

\section{Strengthening contributions and models used}

As mentioned before, several strengthening mechanisms contribute to the yield strength of alloy 718 . The following strengthening contributions and models were used to predict the yield strength of direct aged alloy 718 .

\section{Grain boundary strengthening}

Grain boundary strengthening is a well-known mechanism, which is based on the impeding effect of grain boundaries on the movement of dislocations. It is usually described by the empirical Hall-Petch relation [6,7]. The according grain boundary strengthening contribution $\sigma_{g b}$ can be modeled based on the HallPetch coefficient $k[\mathrm{MPa} \sqrt{\mathrm{m}}]$ and the grain size $d[\mathrm{~m}]$, Equ. 1 .

$$
\sigma_{g b}=k \cdot \frac{1}{\sqrt{d}}
$$

Based on numerous elevated temperature tensile tests of specimens manufactured from different forgings, cf. Fig. 1, the Hall-Petch coefficient was determined to be $0.52 \mathrm{MPa} \sqrt{\mathrm{m}}$ for alloy 718 in both, direct aged (DA) and solution treated and aged (STA) condition.

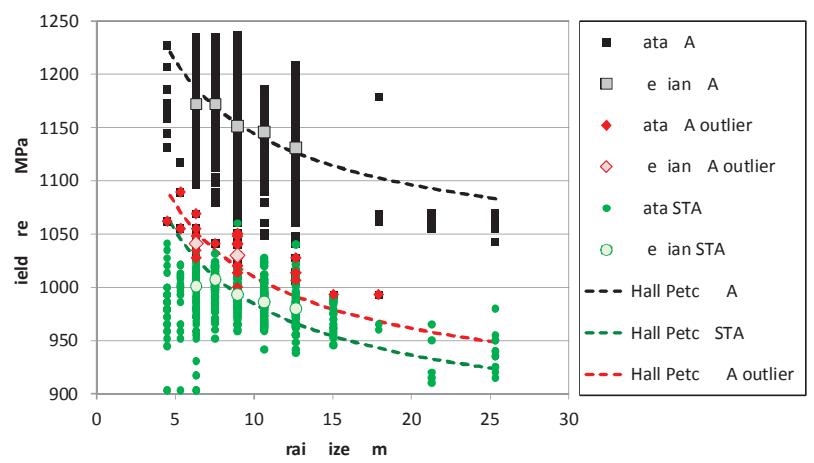

Figure 1. Elevated temperature tensile test results for alloy 718 forgings in direct aged (DA) and solution treated and aged (STA) condition. 
This model is only valid down to a grain size of approximately $7 \mu \mathrm{m}$ (ASTM 11.75), as no further increase in yield strength was found for smaller grain sizes. Outliers were observed for the DA material, exhibiting a $135 \mathrm{MPa}$ lower average yield strength. However, even these outliers seem to follow the proposed HallPetch relation.

\section{Precipitation strengthening}

In general, precipitation strengthening can be caused by shearing and non-shearing mechanisms. For alloy 718 and its $\gamma^{\prime}$ and $\gamma^{\prime \prime}$ precipitates, the coherency effect is predominately responsible for strengthening, cf. [8,9]. Oblak et al. [8] concluded that - while order strengthening ensures the pairwise motion of dislocations the principal obstacle to dislocations is not the ordered particle per se but rather the coherency strain fields of $\gamma^{\prime \prime}$ precipitates. An additional contribution of the antiphase boundary (APB) effect is unclear and is not considered in the present model.

Ahmadi et al. [10] presented equations for the estimation of precipitation strengthening based on physical input parameters such as dislocation character, precipitate radius, outer cut-off radius and mean free distance between precipitates. These models are implemented in the thermo-kinetic software MatCalc, which was used for all precipitation kinetic simulations presented in this paper.

In the case of weak and shearable particles like $\gamma^{\prime}$ and $\gamma^{\prime \prime}$ in alloy 718, Ahmadi et al. [10] assumed that the coherency hardening shear stress $\tau_{C o h}$ correlates with the constrained strain $\varepsilon$ as shown in Equ. 2., where $G$ is the shear modulus of the matrix, $b$ is the magnitude of the Burgers vector in the matrix, $r_{m}$ is the mean radius of the precipitates, $L_{s}$ is the surface to surface distance between two particles, and $T$ is the dislocation line tension.

$$
\tau_{C o h} \sim \sqrt{\frac{G^{3} b \varepsilon^{3} r_{m}^{3}}{L_{s}^{2} T}}
$$

Hence, the modeling of coherency strengthening requires the information of coherency strain, which was taken from literature. Slama et al. $[11,12]$ found that the mismatch parameter for $\gamma^{\prime}$ is very low (around $0.25 \%$ ). For $\gamma^{\prime \prime}$, they reported values lower than $1 \%$ in $<100>$ direction and about $3 \%$ in $<001>$ direction. This means that the coherency strengthening contribution of $\gamma^{\prime}$ is negligible, while it is significant for $\gamma^{\prime \prime}$.

\section{Solid solution strengthening}

Solid solution strengthening in alloy 718 is mainly caused by chromium and molybdenum, cf. [13]. A modeling approach for predicting solid solution strengthening of nickel-based alloys was discussed by Roth et al. [14]. They used the Gypen-Deruyttere model [15], Equ. 3, and presented strengthening constants $k_{i}$ for solid solution strengthening of numerous alloying elements in nickel [16]. Together with the concentration $c_{i}$ of each solute, the yield stress increment $\Delta \sigma$ can be calculated. To be consistent with the dependence on concentration in the Feltham equation [17], Roth et al. [14] suggested to use $n=0.5$.

$$
\Delta \sigma=\left(\sum_{i} k_{i}^{1 / n} c_{i}\right)^{n}
$$

This model is implemented in MatCalc and delivers the solid solution strengthening contribution based on the chemical composition of the matrix. The latter is a result of thermo-kinetic modeling.

\section{Direct age effect}

The conventional heat treatment of alloy 718 (solution treated and aged) includes - after cooling from forging to room temperature a solution annealing at temperatures between $925^{\circ} \mathrm{C}$ and $1000^{\circ} \mathrm{C}$, followed by cooling to room temperature and a duplex aging at $720^{\circ} \mathrm{C}$ and $620^{\circ} \mathrm{C}$. In contrast, direct aging (DA) skips the solution annealing and only includes the duplex aging. Hence, direct aging is the less expensive heat treatment process, but it also has an impact on mechanical properties. On the one hand, a significant increase in yield strength can be observed, cf. Fig. 1, while creep properties remain at an acceptable level. On the other hand, the yield strength scatter increases and even outliers are observed, with yield strength values comparable to the STA condition. This means that the high strength of DA forgings comes along with increased variability. This circumstance highlights the importance of predicting the yield strength of DA forgings for safety critical applications such as engine disks.

The reasons for the significantly higher yield strength of DA material are still not fully understood. Krueger [18] found that the best combination of tensile, creep and low cycle fatigue properties resulted from microstructures with a uniform distribution of fine recrystallized grains and a limited amount of delta phase, which can be achieved in multiple step forging processes [19].

The key to the direct age effect is the need for work hardened microstructures with high dislocation density, cf. [19]. This leads to the assumption that the direct age effect is primarily based on retained warm work in the form of dislocations which are not annealed out in the direct age process.

Jin et al. [20] reported dislocation substructures in direct aged samples, while no substructures were observed in solution annealed samples. Hence, one explanation for the underlying mechanism is that the DA dislocation arrangement leads to increased strain hardening.

Another explanation is that this high dislocation density promotes pipe diffusion and precipitation of $\gamma^{\prime}$ and $\gamma^{\prime \prime}$, which leads to high yield strength due to improved precipitation strengthening. First $3 \mathrm{D}$ atom probe tomography (APT) results of specimens exhibiting a pronounced direct age effect and outliers (which do not show an increased yield strength) revealed no significant difference in $\gamma^{\prime}$ and $\gamma^{\prime \prime}$ precipitation, cf. Fig. 2. In both cases, the fraction of $\gamma^{\prime}$ approximates $7.5 \%$. The fraction of $\gamma^{\prime \prime}$ varies from $16 \%$ in the case of the outlier to $17.5 \%$ in case of the specimen with pronounced direct age effect. No significant differences in precipitate size were determined. The existence of $\gamma^{\prime}-\gamma^{\prime \prime}$-coprecipitates was confirmed.

Nevertheless, further APT analysis will be necessary, as modeling of precipitation strengthening revealed that even small variations of phase fraction and mean radius of $\gamma^{\prime \prime}$ leads to large variations in precipitation strengthening. 

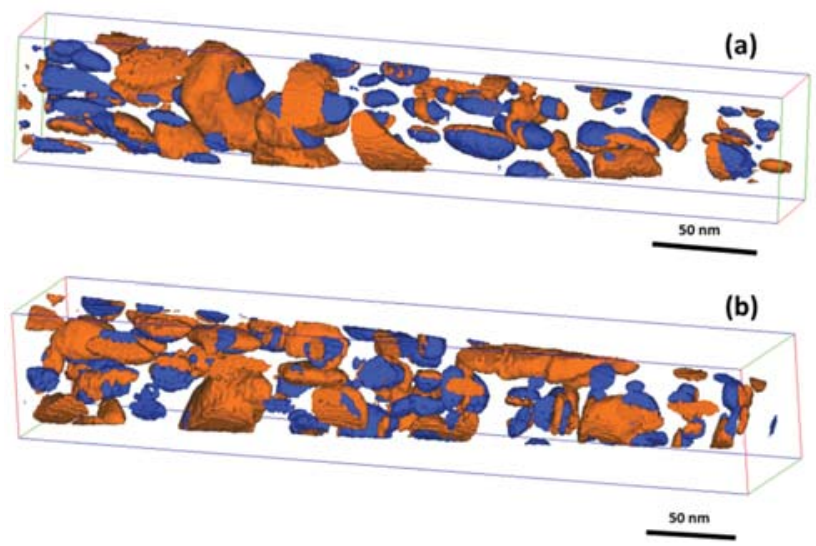

Figure 2. 3D atom probe tomography of specimens exhibiting (a) and not exhibiting (b) a pronounced direct age effect; $\gamma^{\prime}$ precipitates are blue colored and $\gamma^{\prime \prime}$ precipitates and eventually present $\delta$ phase are orange colored.

A third explanation is that the presence of such dislocation arrangements favors the precipitation of certain variants of $\gamma^{\prime \prime}$. Oblak et al. [8] showed that the promotion of the formation of certain variants can lead to significantly higher critical resolved shear stress. Additional transmission electron microscopy analysis will be performed to check this hypothesis.

While models for the evolvement of dislocation density during hot-forming are available, cf. [5, 21], validation is missing due to difficulties in measuring dislocation density of aged alloy 718 . In addition, the modeling of dislocation arrangements causing the direct age effect is currently not possible.

However, as the direct age effect is crucial for modeling the yield strength of direct aged forgings, a phenomenological model was developed. Based on the forming conditions during the last blow, which determine the dislocation arrangement, the so-called Oberwinkler-ratio $O$ was defined. It combines temperature and effective strain, both of which are important in warm working and subsequent property response for direct aged alloy 718 , by relating the present local temperature $T$ in a forging to an effective strain dependent maximum temperature $T_{\max }$ and to an effective strain dependent minimum temperature $T_{\min }$, cf. Equ. 4 to Equ. 6.

The following variables are used in these equations: local effective strain $\varphi$, effective strain coefficients $c_{\max }$ and $c_{\text {min }}$, initial maximum and minimum temperatures $T_{\max 0}$ and $T_{\min 0}$, local forging temperature $T$ and Oberwinkler-ratio $O$. Equ. 6 is only valid if the condition $\varphi_{\min } \leq \varphi \leq \varphi_{\max }$ is fulfilled. Per definition, $O=-\infty$ if $\varphi<\varphi_{\min }$ and $O=+\infty$ if $\varphi>\varphi_{\max }$.

$$
\begin{gathered}
T_{\max }=T_{\max 0}-c_{\max } \varphi^{2} \\
T_{\min }=T_{\min 0}-c_{\min } \varphi^{2} \\
O=\frac{T-T_{\min }}{T_{\max }-T_{\min }}
\end{gathered}
$$

Hence, the Oberwinkler-ratio compares the actual process conditions (temperature, effective strain) with a nominal process window for optimum direct age effect.
This nominal process window is defined in terms of an effective strain dependent upper and lower temperature limit $\left(T_{\min }\right.$ and $\left.T_{\max }\right)$ and upper and lower limits for effective strain $\left(\varphi_{\min }\right.$ and $\left.\varphi_{\max }\right)$, by conducting multiple forgings at a range of temperatures and with a range of local effective strain values, observing where acceptable direct age strengthening is achieved, and fitting the process parameters for those forgings into the form shown in Equ. 6.

The exact $T_{\min }$ and $T_{\max }$ values are somewhat dependent on the die practice used, but the temperature range for alloy 718 typically lies between $900{ }^{\circ} \mathrm{C}$ and $1010^{\circ} \mathrm{C}$ and effective strain values above 0.02 have been found to be needed.

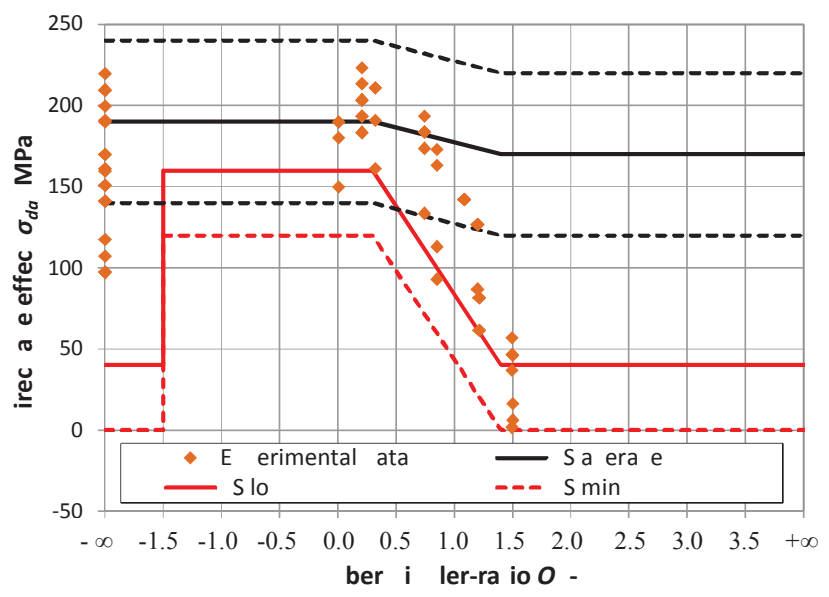

Figure 3. Correlation of the direct age effect $\sigma_{d a}$ and Oberwinklerratio $O$ and exemplarily comparison with experimental data of a forging sampled in multiple locations having unique Oberwinklerratio values according to their particular process conditions; YS average describes the average expected yield strength, YS low the average yield strength of outliers and YS $\min$ the minimum expected yield strength.

Fig. 3 exemplarily shows experimental data of a direct aged forging. Thereby, Oberwinkler-ratio $-\infty$ means that the effective strain at the specimen position is too low to guarantee the full direct age effect $\left(\varphi<\varphi_{\min }\right)$, whereas $+\infty$ indicates - at the present process temperature - too much effective strain for an optimum direct age effect. Phenomenological modeling is based on the lines shown in Fig. 3.

Certain forming conditions (Oberwinkler-ratio $>0.3$ ) foster the occurrence of outliers with low or - in the worst case - no direct age effect. This is the reason for outliers with very low yield strength as observed in Fig. 1. The underlying mechanism is not fully understood. However, it is believed that recrystallization will be the crucial factor.

Furthermore, an impact of feedstock was determined, both, on the probability of occurrence and magnitude of the yield strength of outliers. This feedstock inhomogeneity seems to be the reason for outliers in circumferential direction of rotational symmetric forgings, which were determined during this extensive testing program. Further investigation of the potential factors behind these outliers is being made. 
Basic yield strength and strain hardening

Strain hardening was not considered separately. This was done due to the fact that dislocation density can hardly be measured experimentally in alloy 718 , which means lacking data for model calibration. In addition, strain hardening and the direct age effect are interrelated. Hence, basic yield strength and strain hardening were summed up and determined together by subtracting the modeled yield strength contributions of grain boundary, precipitation and solid solution strengthening as well as the direct age effect from experimentally determined yield strength values.

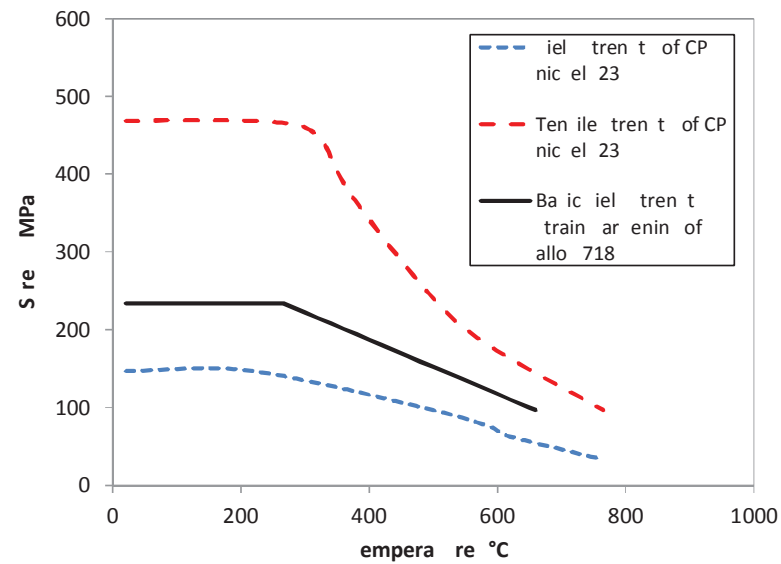

Figure 4. Comparison of temperature dependence of tensile test results for commercially pure nickel according to [22] and basic yield strength plus strain hardening of alloy 718 .

The result - a temperature dependent basic yield strength including the expected strain hardening contribution after solution annealing - was compared with the yield strength of commercially pure (CP) wrought nickel [22], Fig. 4. The temperature dependence of basic yield strength and the yield strength of CP nickel are in good accordance. The basic yield strength of CP nickel can be estimated on the basis of results of Park and Parker [23], who reported Hall-Petch plots for CP nickel. Their results indicate that grain boundary strengthening of $\mathrm{CP}$ nickel is comparable with those of alloy 718 , leading to a roughly estimated basic yield strength of only $10 \mathrm{MPa}$.

The resulting strain hardening of $230 \mathrm{MPa}$ corresponds according to the model implemented in MatCalc [21] - to a dislocation density of $8 \mathrm{E} 13 \mathrm{~m}^{-2}$. This seems to be a realistic magnitude for solution annealed material, as Jin et al. [20] reported dislocation densities higher than $1 \mathrm{E} 15 \mathrm{~m}^{-2}$ for the direct aged condition.

\section{$\underline{\text { Relative importance of strengthening contributions }}$}

The total yield strength $\sigma_{y}$ is derived by linearly combining [24, 25] the six strengthening contributions: basic yield strength $\sigma_{b}$ plus strain hardening $\sigma_{s h}$, grain boundary strengthening $\sigma_{g b}$, precipitation strengthening $\sigma_{p}$, solid solution strengthening $\sigma_{s s}$, and the direct age effect $\sigma_{d a}$, Equ. 7 .

$$
\sigma_{y}=\sigma_{b}+\sigma_{s h}+\sigma_{g b}+\sigma_{p}+\sigma_{s s}+\sigma_{d a}
$$

With respect to the relative importance of the different strengthening contributions, the following rough quantities (typical variations within a forging in brackets) were determined based on modeling and experimental results for room temperature testing:

$$
\begin{array}{ll}
\text { Basic yield strength of nickel } & 10 \mathrm{MPa} \\
\text { + strain hardening } & +230 \mathrm{MPa} \\
\text { + grain boundary strengthening } & +150 \mathrm{MPa}( \pm 20 \mathrm{MPa}) \\
\text { + precipitation strengthening } & +520 \mathrm{MPa}( \pm 50 \mathrm{MPa}) \\
\text { + solid solution strengthening } & +260 \mathrm{MPa} \\
\text { + direct age effect } & +180 \mathrm{MPa}(+10 /-140 \mathrm{MPa}) \\
\hline \text { Total yield strength } & 1350 \mathrm{MPa}
\end{array}
$$

This evaluation highlights the importance of precipitation strengthening (the largest single yield strength contribution) and direct age effect (yield strength contribution with the - by far largest variation).

\section{Precipitation modeling}

Thermo-kinetic modeling was performed with MatCalc, cf. [26], using the thermodynamic database mc_ni_v2.029.tdb and the mobility database mc ni_v2.005.ddb. In general, considered precipitates in alloy $7 \overline{18}$ are carbides and carbonitrides, Laves phase, $\delta$ phase, $\gamma^{\prime}$ and $\gamma^{\prime \prime}$.

As Laves phase is completely dissolved during feedstock homogenization and, hence, not present in billets, it is not considered during modeling of the forging process to increase computational efficiency. Modeling results are the according number densities, phase fractions and mean radii of all precipitates, cf. Fig. 5 .

To achieve meaningful output from thermo-kinetic modeling, a sound calibration of undetermined precipitation and strengthening modeling parameters is necessary. Two different methods were used for calibration. On the one hand, results of tensile tests in combination with literature information as discussed in [27] were used. On the other hand, yield strength values of multiple positions within several different direct aged forgings in combination with 3D atom probe tomography and scanning electron microscopy (for characterization of precipitates) were taken as a reference.

\section{Integrated process modeling}

At Böhler Schmiedetechnik $\mathrm{GmbH}$ \& $\mathrm{Co} \mathrm{KG}$ (Austria) the simulation of the thermo-mechanical processing is performed with the finite element software DEFORM ${ }^{\mathrm{TM}}$. The average grain size distribution in a forging is also modeled in DEFORMTM , based on a user sub routine with underlying semi-empirical models [3]. Similarly, the Oberwinkler-ratio is calculated with a user sub routine. A point tracking can be performed for each specimen position. The generated history data (temperature vs. time, strain rate vs. time, grain size vs. time, etc.) of the whole production process is used as an input for thermo-kinetic precipitation modeling, Fig. 6. 

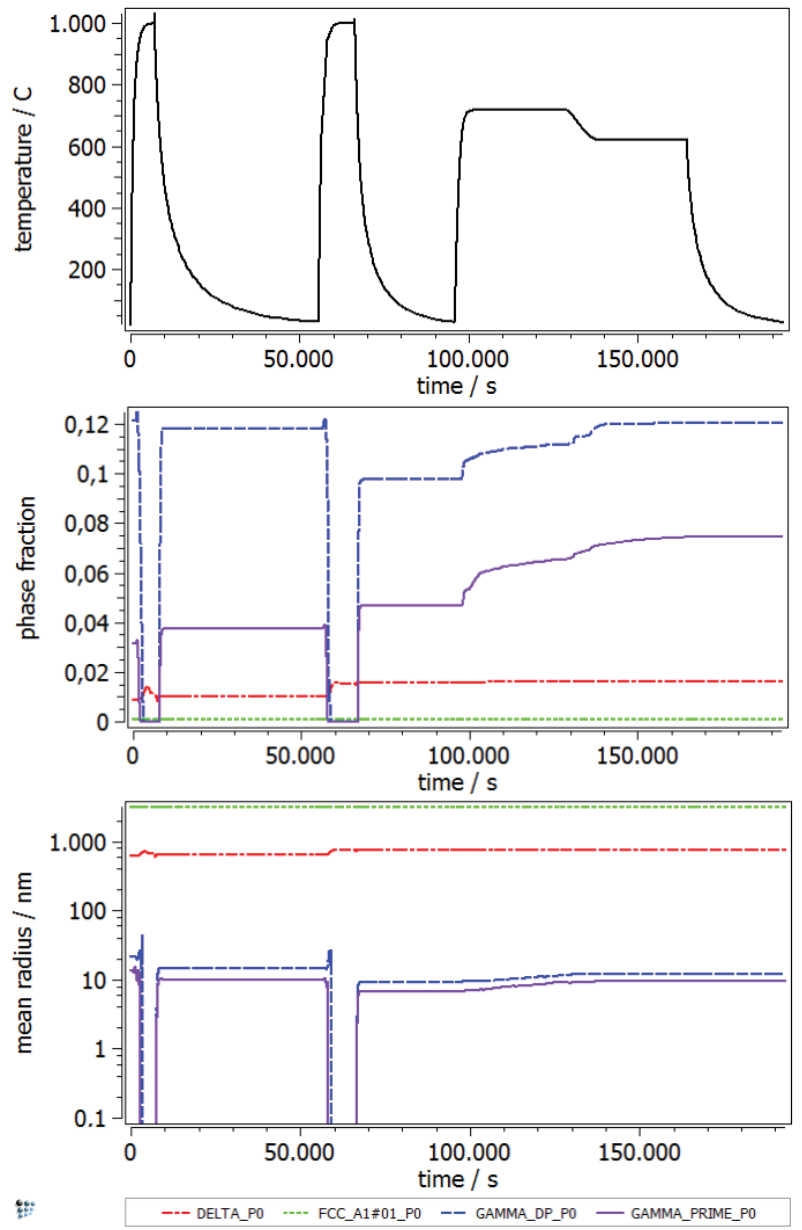

Figure 5. Thermo-kinetic precipitation modeling of thermomechanical processing of a direct aged forging in MatCalc: temperature vs. time as an input, resulting phase fraction and mean radius history as an output.
As mentioned above, thermo-kinetic modeling is performed with MatCalc. It starts with a Scheil calculation for determination of phase fractions at solidification of the vacuum arc remelting (VAR) ingot. This is the starting point for precipitation simulation of air cooling after solidification, subsequent homogenization treatment at temperatures well above $1000{ }^{\circ} \mathrm{C}$ and finally ingot breakdown and GFM converting. This feedstock information is saved in a binary file, which serves as a basis for precipitation modeling of the forging process.

The thermo-kinetic modeling of the forging process and subsequent heat treatments incorporates the following steps: loading of the binary file of the feedstock billet, loading of history data (temperature vs. time, grain size vs. time, etc.) generated with DEFORM $^{\mathrm{TM}}$ during a point tracking, precipitation modeling based on this input and export of relevant data after completion of precipitation modeling. For enabling efficient computing of several points within a forging, this procedure is automated by batch processing.

For each chosen point tracking position, four additional point tracking positions are defined within a radius of $3 \mathrm{~mm}$ to reduce the influence of numerical oscillations due to MatCalc's Kampmann-Wagner approach [26]. The results of all five points are finally averaged during post-processing.

After completion of thermo-mechanical and thermo-kinetic simulation, the information is processed in a tool called Bohler Mechanical Properties Postprocessor (BoMePP), cf. Fig. 6. With this software, yield strength contributions and the total yield strength can be calculated.

Thereby, solid solution and precipitation strengthening are imported results of MatCalc, grain boundary strengthening and the direct age effect are modeled based on results of the DEFORM $^{\mathrm{TM}}$ simulation as explained above, and the basic yield strength is determined in accordance with Fig. 4.
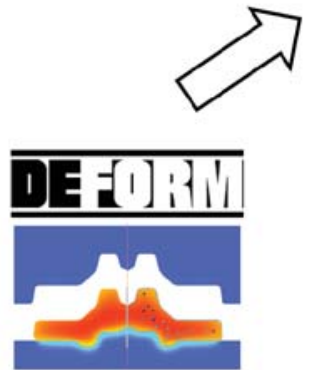

- Temperature vs. time

- Effective strain vs. time

- Effective strain rate vs. time

- Grain size vs. time

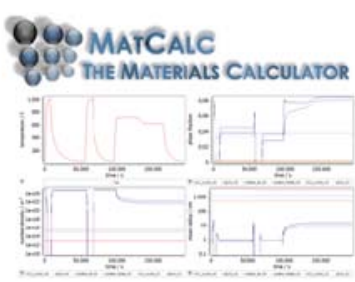

- Phase fractions vs. time

- Precipitate sizes vs. time

- Yield strength contributions vs. time
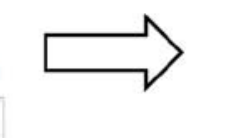

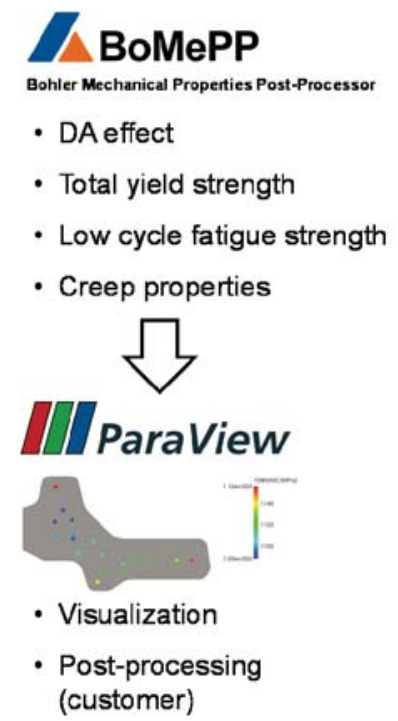

Figure 6. Overview of the integrated process modeling procedure at Böhler Schmiedetechnik GmbH \& Co KG. (DEFORM ${ }^{\mathrm{TM}}$ is a registered trademark of Scientific Forming Technologies Corporation) 


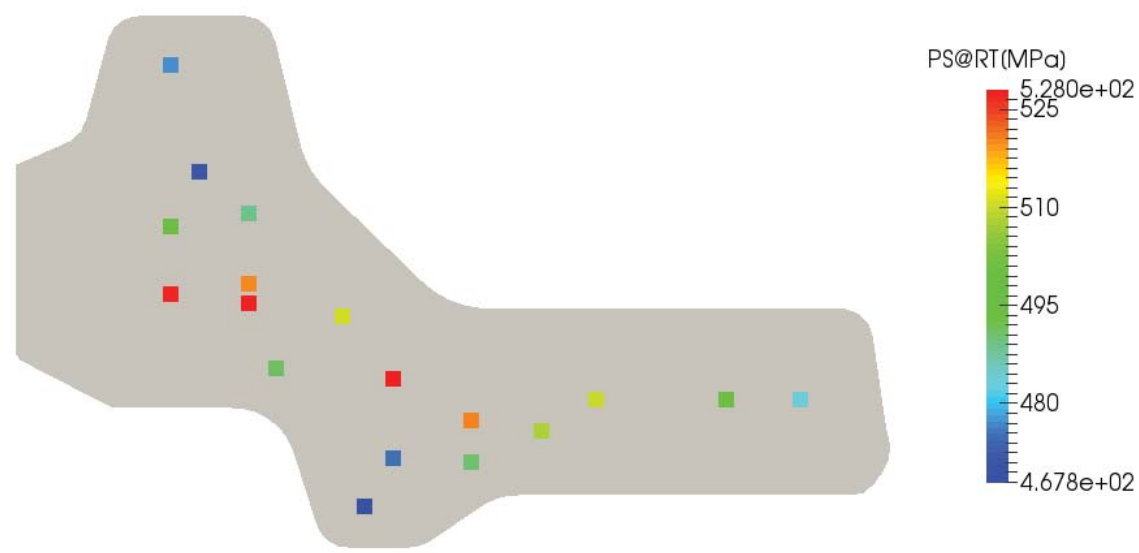

Figure 7. Graphical representation of the precipitation strengthening contribution in a direct aged engine disk. Similar plots are made for all strengthening contributions, and the individual contributions are summed to model the total strength for the part.

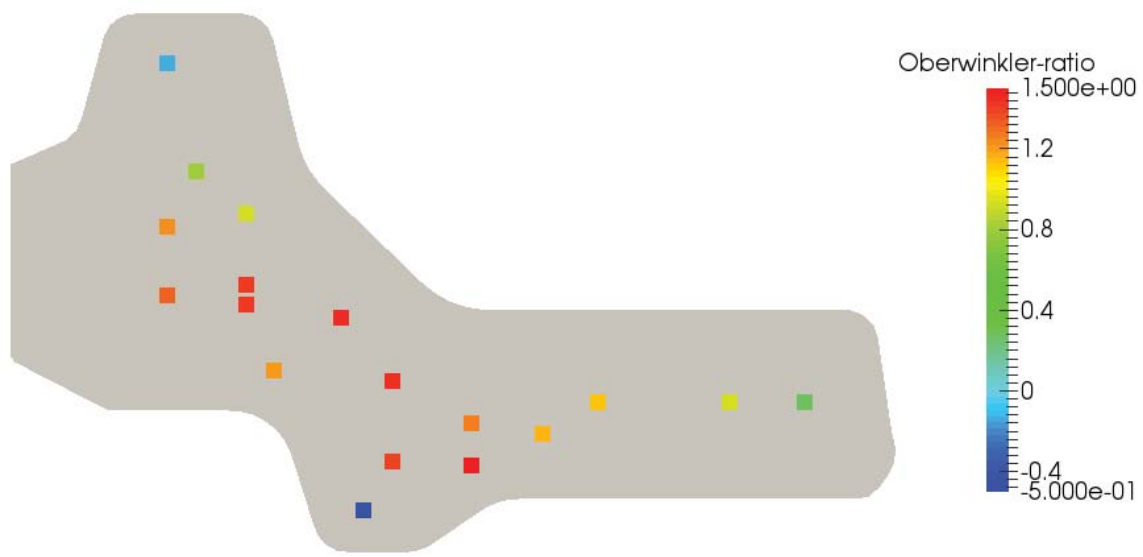

Figure 8. Graphical representation of the Oberwinkler-ratio distribution in a direct aged engine disk.

Furthermore, models for predicting creep and low cycle fatigue behavior are included in BoMePP. Low cycle fatigue modeling is based on the Manson-Coffin-Basquin equations under consideration of grain size, yield strength and testing temperature, cf. [28].

The minimum creep rate $\dot{\varepsilon}_{\min }$ is modeled under consideration of grain size, precipitates, testing temperature as well as stress, by combining models from Han and Chaturvedi [29] and Oruganti et al. [30], cf. Equ. 8. The first row of Equ. 8 describes the influence of precipitates, stress and temperature [30] by using the following variables: fitting constant $A$, activation energy $Q_{c}$ $(460,000 \mathrm{~J} / \mathrm{mol})$, gas constant $R(8.314 \mathrm{~J} / \mathrm{mol} \mathrm{K})$, temperature $T$ [K], stress $\sigma$ [MPa], phase fraction $f$ of $\gamma^{\prime}$ and $\gamma^{\prime \prime}$ precipitates [-], and exponent $n(0.68)$.

The second row of Equ. 8 considers the influence of grain size [29] and exhibits the following variables: fraction of diffusional creep $f_{D}$, Burgers vector $b(2.5 \mathrm{E}-10 \mathrm{~m})$, average grain size $d[\mathrm{~m}]$, diffusional creep exponent $x(0.19)$, and dislocation power law creep exponent $y(-0.42)$.

$$
\begin{aligned}
\dot{\varepsilon}_{\min }= & A \exp \left(-\frac{Q_{C}}{R T}\right) \sinh \left([\sigma-f \sigma]^{n}\right) \\
& \cdot\left(f_{D}\left(\frac{b}{d}\right)^{x}+\left(1-f_{D}\right)\left(\frac{b}{d}\right)^{y}\right)
\end{aligned}
$$

In addition, the Oberwinkler-ratio has also a considerable influence on the creep behavior and must not be neglected. While for an optimum yield strength an Oberwinkler-ratio of $<0.3$ is desirable, cf. Fig. 3, lowest creep rates were observed for an Oberwinkler-ratio $>1$, Fig. 9. This means that those mechanisms leading to the direct age effect and increased yield strength also increase the minimum creep rate - at least for testing conditions in the transition region [29] between diffusional and power law creep.

The output of BoMePP is a vtk-file, which can be opened with ParaView (an open-source software, which eases sharing with customers) and enables the graphical representation of the modeling results, cf. Fig. 7 and Fig. 8. 


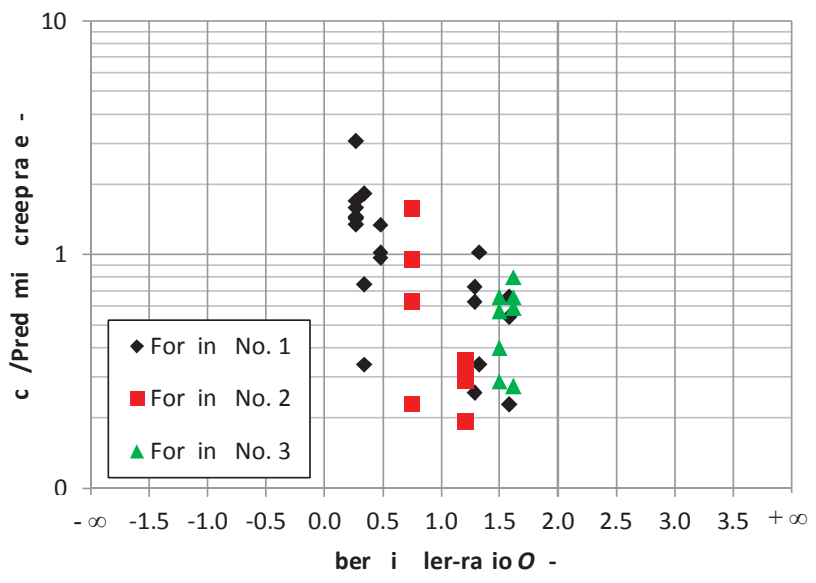

Figure 9. Ratio of actual and predicted minimum creep rate plotted against the Oberwinkler-ratio $O$. Predicted minimum creep rate according to Equ. 8. The forgings were sampled in multiple locations having unique Oberwinkler-ratio values according to their particular direct age blow conditions.

The computational time necessary for modeling mechanical properties of engine disks comprises of approximately 20 minutes for the DEFORM ${ }^{\mathrm{TM}}$ 2D simulation and 6 minutes per MatCalc simulation (each specimen position requires five MatCalc simulations). Hence, a precipitation simulation for 18 positions as shown in Fig. 7 can be done overnight in about 9 hours.

\section{Verification}

The results of the thermo-kinetic modeling like phase fractions of $\gamma^{\prime}$ (ca. 8\%), $\gamma^{\prime \prime}$ (ca. 13\%) and delta phase (ca. 2 - 5\%) were compared with light optical microscopy and 3D atom probe tomography results. A good agreement was observed. Verification of the yield strength was achieved by comparing experimental cut-up results of several forgings and the according modeling results, cf. Fig. 10. A good correlation was observed in all cases. Especially those positions with potential outliers with low yield strength due to variations of the direct age effect (e.g. specimen positions 10 and 15 - due to their high Oberwinkler-ratio of 1.5) were identified correctly. This confirms the validity of the chosen modeling approach.

The minimum yield strengths of those positions with an Oberwinkler-ratio of $-\infty$ are underestimated in the case presented in Fig. 10. This means that the chosen minimum effective strain $\varphi_{\min }$ for a fully developed direct age effect might be overconservative.

\section{Optimization}

The verified integrated process modeling approach was used for optimization of the forging process of a direct aged engine disk. The existing process led to cut-up tensile test results near or below the yield strength specification, Fig. 11.

Modeling confirmed that the chosen forging parameters caused an Oberwinkler-ratio $>0.3$ in certain specimen positions and, hence, an imperfect direct age effect corresponding to low strength.

Optimization of the forging process in terms of temperature and effective strain per blow as well as Oberwinkler-ratio was achieved by varying process parameters (such as, for example, die displacements) during process modeling. The resulting optimized forging process was implemented and cut-up results confirmed a $9 \%$ increase of the minimum yield strength (well above the specification limit) and a much more homogeneous yield strength distribution with less scatter, Fig. 12.

Fig. 10 to 12 show positions within typical production forgings currently in manufacture. Although the forging shapes cannot be shown, the test positions were sampled across the forging shapes and show that the Oberwinkler-ratio has a reasonable correlation to properties. Correlation is better in some regions of the forgings than in others, highlighting the complexity and multiple factors involved in warm working and subsequent heat treatment response.

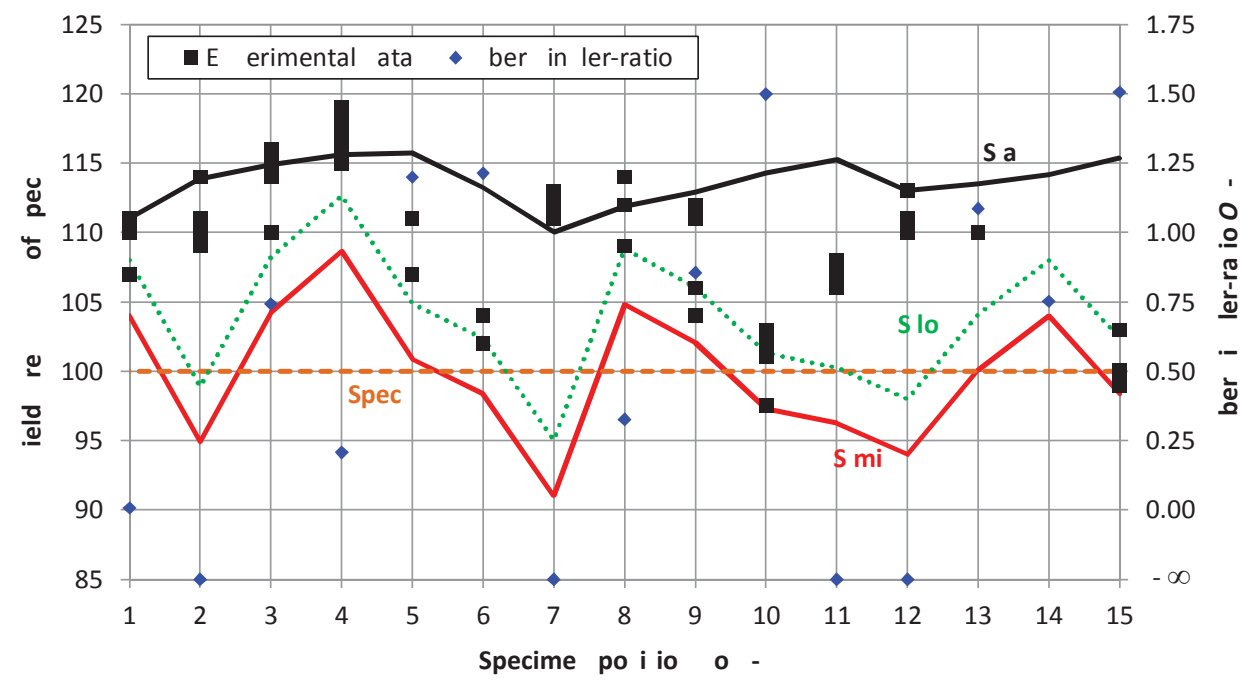

Figure 10. Comparison of elevated temperature tensile test data (black squares) and modeling results (lines) of a direct aged engine disk. In addition, the Oberwinkler-ratio is plotted (blue diamonds). 


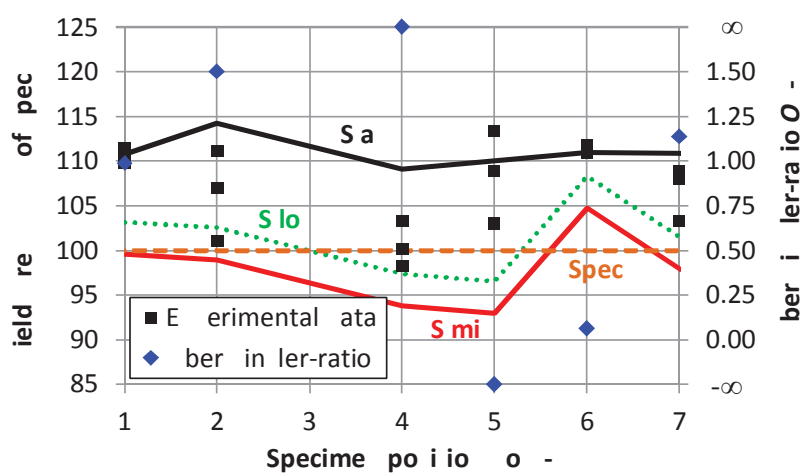

Figure 11. Comparison of elevated temperature tensile test data (black squares) and modeling results (lines) of a direct aged engine disk with imperfect direct age blow conditions. In addition, the Oberwinkler-ratio is plotted (blue diamonds). No elevated tensile test data were obtained at position 3 .

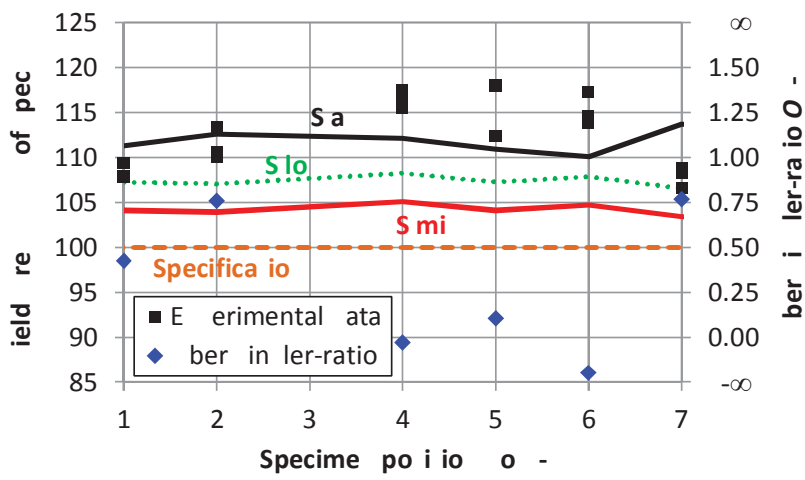

Figure 12. Comparison of elevated temperature tensile test data (black squares) and modeling results (lines) of an optimized direct aged engine disk with optimum direct age blow conditions. In addition, the Oberwinkler-ratio is plotted (blue diamonds). No elevated tensile test data were obtained at position 3 .

\section{Conclusion}

An evaluation of the relative importance of strengthening contributions revealed the importance of precipitation strengthening (the largest single yield strength contribution) and direct age effect (yield strength contribution with the - by far largest variation). The key to a reliable prediction of precipitation strengthening is a sound thermo-kinetic precipitation modeling, which was achieved in MatCalc by thorough calibration.

The direct age effect was described by a phenomenological model (the Oberwinkler-ratio), which is capable to identify those regions of a forging, where the direct age effect is not fully evolved, leading to outliers with significantly lower yield strength. Further investigation of the potential factors behind these outliers is being made.

At Böhler Schmiedetechnik $\mathrm{GmbH}$ \& $\mathrm{Co} \mathrm{KG}$, an integrated process modeling procedure was developed, which is able to predict mechanical properties of direct aged alloy 718 forgings.
It includes thermo-mechanical process modeling in DEFORM ${ }^{\mathrm{TM}}$, thermo-kinetic precipitation modeling in MatCalc, mechanical properties post-processing in BoMePP and visualization in ParaView.

Upfront prediction of mechanical properties leads to unique benefits for both, customer and forging house. On the one hand, the thermo-mechanical processing can be optimized in terms of processing parameters (such as, for example, die displacements) and disk shape to achieve the highest strength in the most critical regions of an engine disk. On the other hand, the influencing process parameters with respect to yield strength and other mechanical properties can be identified and used to achieve a robust manufacturing process.

\section{Acknowledgements}

This project has received funding from the Clean Sky Joint Undertaking under the European Union's Seventh Framework Programme under grant agreement No 296526, and it has received funding from the Clean Sky 2 Joint Undertaking under the European Union's Horizon 2020 research and innovation programme under grant agreement No 714043.

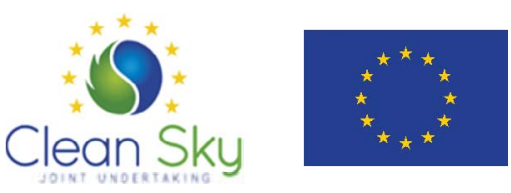

\section{References}

1. D.F. Paulonis and J.J. Schirra, "Alloy 718 at Pratt \& Whitney Historical Perspective and Future Challenges," Superalloys 718 , 625, 706 and Various Derivatives, ed. E.A. Loria (Warrendale, PA: The Minerals, Metals \& Materials Society, 2001), 13-23.

2. J.A. Heaney et al., "Development of a New Cast and Wrought Alloy (René 65) for High Temperature Disk Applications," $8^{\text {th }}$ Int. Symposium on Superalloy 718 and Derivatives, eds. E. Ott et al. (Hoboken, NJ: Wiley, 2014), 67-77.

3. M. Stockinger, "Mikrostrukturelle Simulation des Gesenkschmiedens von Nickelbasis-Legierungen [Microstructural Simulation of the Die Forging Process of Nickel-Based Alloys]" (Ph.D. thesis, TU Graz, 2003).

4. D. Furrer et al., "Computational Modeling and Simulation of Alloy 718," $8^{\text {th }}$ Int. Symposium on Superalloy 718 and Derivatives, eds. E. Ott et al. (Hoboken, NJ: Wiley, 2014), 79-94.

5. B. Marty et al., "Recrystallization and Work-Hardening Prediction During Forging Process of Inconel 718," Superalloys $718,625,706$ and Various Derivatives, ed. E.A. Loria (Warrendale, PA: The Minerals, Metals \& Materials Society, 1997), 331-342.

6. E.O. Hall, "The Deformation and Ageing of Mild Steel: III. Discussion of Results," Proc. Phys. Soc., B64 (1951), 747-753. 
7. N.J. Petch, "The Cleavage Strength of Polycrystals," J. Iron Steel Inst., 174 (1953), 25-28.

8. J.M. Oblak, D.F. Paulonis and D.S. Duvall, "Coherency Strengthening in $\mathrm{Ni}$ Base Alloys Hardened by $\mathrm{DO}_{22} \gamma^{\text {" }}$ Precipitates," Metallurgical Transactions, 5 (1974), 143-153.

9. M.C. Chaturvedi and Y. Han, "Strengthening Mechanisms in Inconcel 718 Superalloy," Metal Science, 17 (1983), 145-149.

10. M.R. Ahmadi et al., "A Model for Precipitation Strengthening in Multi-Particle Systems," Comp. Materials Sci., 91 (2014), 173186.

11. C. Slama, C. Servant and G. Cizeron, "Aging of the Inconel 718 Alloy Between 500 and $750{ }^{\circ} \mathrm{C}$," Journal of Materials Research, 12 (1997), 2298-2316.

12. C. Slama and M. Abdellaoui, "Structural Characterization of the Aged Inconel 718," Journal of Alloys and Compounds, 306 (2000), 277-284

13. C.T. Sims, N.S. Stoloff and W.C. Hagel, Superalloys II (New York, NY: John Wiley \& Sons, Inc., 1987), 63.

14. H.A. Roth, C.L. Davis and R.C. Thomson, "Modeling Solid Solution Strengthening in Nickel Alloys," Metallurgical and Materials Trans. A, 28 (1997), 1329-1335.

15. L.A. Gypen and A. Deruyttere, "Multi-Component Solid Solution Hardening," Journal of Materials Science, 12 (1977), 1028-1033.

16. Y. Mishima et al., "Solid Solution Hardening of Nickel - Role of Transition Metal and B-Subgroup Solutes," Transactions of the Japan Institute of Metals, 27 (1986), 656-664.

17. P. Feltham, "Solid Solution Hardening of Metal Crystals," Journal of Physics D: Applied Physics, 1 (1968), 303-308.

18. D.D. Krueger, "The Development of Direct Age 718 for Gas Turbine Engine Disk Applications," Superalloy 718 - Metallurgy and Applications, ed. E.A. Loria (Warrendale, PA: The Minerals, Metals \& Materials Society, 1989), 279-296.

19. W. Horvath et al., "The Effectiveness of Direct Aging on Inconel 718 Forgings Produced at High Strain Rates as Obtained on a Screw Press," Superalloys 718, 625, 706 and Various Derivatives, ed. E.A. Loria (Warrendale, PA: The Minerals, Metals \& Materials Society, 2001), 223-228.

20. Z. Jin, G. Jiang and R. Zhou, "The Influence of Direct Aging Treatment on the Structure and the Mechanical Properties for Inconcel-718 Alloy," High Temperature Alloys for Gas Turbines and Other Applications 1986 - Part II, eds. W. Betz et al. (Dordrecht, Holland: D. Reidel Publishing Company, 1986), 921932

21. P. Sherstnev, P. Lang and E. Kozeschnik, "Treatment of Simultaneous Deformation and Solid-State Precipitation in Thermo-Kinetic Calculations," ECCOMAS 2012 - European Congress on Computational Methods in Applied Sciences and
Engineering, eds. J. Eberhardsteiner et al. (Vienna, Austria: Vienna University of Technology, 2012), 5331-5338.

22. Special Metals Corporation, "Nickel 200 \& 201," Retrieved January 19， 2016 from http://www.specialmetals.com/assets/ documents/ alloys/nickel-duranickel/nickel-200-201.pdf.

23. N.K. Park and B.A. Parker, "Effect of Grain Size on the Deformation Behaviour of Nickel and Nickel Alloy Sheet," Strength of Metals and Alloys Vol. 3, eds. H.J. McQueen et al. (Oxford, UK: Pergamon Press, 1986), 159-164.

24. O.R. Myhr, Ø. Grong and S.J. Andersen, "Modelling of the Age Hardening Behaviour of Al-Mg-Si alloys," Acta Materialia, 49 (2001), 65-75.

25. R. Schnitzer, S. Zinner and H. Leitner, "Modeling of the Yield Strength of a Stainless Maraging Steel," Scripta Materialia, 62 (2010), 286-289

26. E. Kozeschnik, Modeling Solid-State Precipitation (New York, NY: Momentum Press, LLC, 2013), 383-399.

27. A. Drexler et al., "Yield Stress Evolution in Inconel 718 Samples Under Standard Heat Treatment Process Conditions of Turbine Disks," CD-ROM proceedings of the European Conference on Heat Treatment 2015 and $22^{\text {nd }}$ IFHTSE Congress, (Milan, Italy: Associazione Italiana di Metallurgia, 2015).

28. H. Maderbacher et al., "The Influence of Microstructure and Operating Temperature on the Fatigue Endurance of Hot Forged Inconel 718 Components," Materials Science \& Engineering A, 585 (2013), 123-131.

29. Y. Han and M.C. Chaturvedi, "Steady State Creep Rate Equation of Inconel 718 Superalloy," Chin. J. Met. Sci. Technol., 5 (1989), 79-84.

30. R. Oruganti et al., "A New Approach to Modeling of Creep in Superalloys," Superalloys 2012, eds. E. Huron et al. (Hoboken, NJ: Wiley, 2012), 473-479. 